\title{
Regression of a Congenital Mesoblastic Nephroma
}

\author{
Sarah Whittle ${ }^{1}$, Ankush Gosain, MD ${ }^{1}$, Petagay Y. Scott Brown, MD², Lairsa Debelenko, MD, \\ $\mathrm{PhD}^{3}$, Susana Raimondi, $\mathrm{PhD}^{3}$, Judith A. Wilimas, MD ${ }^{4}$, Jesse J. Jenkins, MD ${ }^{3}$, and \\ Andrew M. Davidoff, MD ${ }^{1,}{ }^{*}$ \\ ${ }^{1}$ Department of Surgery, St. Jude Children's Research Hospital, Memphis, TN \\ 2 Department of Pediatrics, Bustamante Hospital for Children, Kingston, Jamaica \\ 3 Department of Pathology, St. Jude Children's Research Hospital, Memphis, TN \\ ${ }^{4}$ Department of Oncology, St. Jude Children's Research Hospital, Memphis, TN
}

\section{Abstract}

Histologically, the cellular variant of congenital mesoblastic nephroma (CMN) is very similar to another rare tumor of infancy, infantile fibrosarcoma (IFS). In addition to the histologic similarities, these tumor types share cytogenetic abnormalities including translocation $\mathrm{t}(12 ; 15)$ (p13;q25). We describe herein the case of a child who did not have immediate surgical resection of a CMN and whose tumor was untreated for 8 months. During that time, the tumor demonstrated a significant degree of regression. The shared translocation with IFS, a tumor with welldocumented potential for spontaneous regression, suggests that this genetic abnormality may have contributed to the favorable clinical course.

\section{Keywords}

congenital mesoblastic nephroma; translocation; ETV6; regression

\section{INTRODUCTION}

Nearly two-thirds of abdominal masses in infants are of renal origin, with most being congenital abnormalities (mostly cystic renal disease and hydronephrosis). Of the renal neoplasms in neonates and infants under 3 months of age, congenital mesoblastic nephroma $(\mathrm{CMN})$ is the most common, and, overall, comprises about $3-10 \%$ of all childhood renal tumors.[1] Mesoblastic nephroma may be detected on routine prenatal ultrasonography or may present as a palpable abdominal mass in a young infant. CMN is divided into two histologic subtypes: classic and cellular. The classic histiotype, first distinguished from Wilms tumor by Bolande in 1967, is characterized by leiomyomatous histology with spindle cells in bundles, rare mitoses and the absence of necrosis.[2] The cellular subtype, subsequently described by Joshi in 1986, has a more cellular appearance, higher mitotic index, and often has areas of necrosis.[3] The biologic behavior of CMN is generally benign, [4] although rare cases of local recurrence and metastasis have been reported, usually with the cellular subtype.[5] Nevertheless, surgical resection with nephrectomy is considered adequate therapy for both subtypes, if complete resection is achieved. A retrospective review of renal tumors in children in the first 7 months of life by van den Heuvel-Eibrink reported 5-year event free survival (EFS) of 94\%, and overall survival (OS) of $96 \%$ for

\footnotetext{
*Correspondence to: Andrew Davidoff, MD, Department of Surgery, St. Jude Children's Research Hospital, 262 Danny Thomas Place, Memphis, TN 38105-3678, Tel: 901-5959-4060, andrew.davidoff@stjude.org.
} 
infants with CMN.[6] Furtwaengler reported similar overall survival results, further noting that any recurrence and/or death occurred only in patients with the cellular subtype, whose EFS and OS rates were $85 \%$ and $90 \%$, respectively.[5]

We describe a patient with CMN that was felt to be unresectable at birth. However, despite receiving no adjuvant therapy, the mass showed evidence of regression over a period of eight months.

\section{CASE REPORT}

An 8 month-old female was transferred to our institution with a left congenital mesoblastic nephroma, present since birth. Within minutes after birth, the patient had been noted to have progressively worsening abdominal distention. A plain radiograph revealed a shift of the bowel to the right of the abdomen with significant mass effect on the left. Abdominal computed tomography $(\mathrm{CT})$ revealed a $9.2 \times 8.8 \times 11.5 \mathrm{~cm}$ solid mass without obvious necrosis, fluid or calcification, originating from the left kidney, displacing the bowel, aorta and inferior vena cava (IVC) to the right (Figure 1A). There was no evidence of disseminated disease on chest CT. Neither hypertension nor hypercalcemia were present. The patient was hospitalized and received supportive care for two months, with the hospital course being complicated by an enterococcal urinary tract infection. Ultimately, the patient underwent an exploratory laparotomy at 3 months of age. At surgery, the left renal tumor was noted to infiltrate the mesentery of the splenic flexure and descending colon; the IVC and aorta were not visualized. Due to the extent of tumor, it was deemed unresectable by the operating surgeon. A small incisional biopsy showed a primitive spindle cell tumor (Figure 1B), positive for muscle-specific actin and vimentin by immunohistochemistry. The proliferative index was approximately 15-20\%, as scored by the percentage of Ki67-positive cells (Figure 1C). Interphase in situ hybridization performed on formalin-fixed, paraffinembedded tumor sections using an ETV6 DNA probe (Dako, Denmark) showed split signals, suggesting rearrangement of the ETV6 gene locus in $48 \%$ of tumor nuclei (Figure 1D). Thus, the clinical presentation, morphology and cytogenetics were diagnostic of a cellular variant of congenital mesoblastic nephroma. Three weeks after surgical exploration, the patient's clinical course was complicated by bacterial meningitis. She was discharged from her local hospital after recovering from that infection.

The child was referred to our institution for further management of her renal tumor when she was 8 months of age. Computed tomography obtained upon arrival demonstrated significant reduction in the size of the left intrarenal mass, to $7.2 \times 5.7 \times 5.4 \mathrm{~cm}$. In addition, there were large areas of intratumoral fluid and calcification not seen on initial imaging (Figure 2A). Again, no evidence of metastases was noted. Shortly thereafter, the patient underwent an uncomplicated left nephrectomy. Gross examination of the specimen revealed an enlarged kidney that weighted $160 \mathrm{gm}$ and contained an irregularly shaped, $7 \mathrm{~cm}$ predominantly cystic mass that occupied the lower pole of the kidney (Figure 2B). The tumor involved the renal sinus, and grew outside of the renal capsule and into the perirenal adipose tissue. The mass had significant cystic degeneration with less than $10 \%$ viable tumor. Microscopically, the central area of necrosis showed signs of organized hematoma with hemosiderosis, calcifications and scarring (Figure 2C). Residual viable tumor found at the periphery of the cystic mass had morphology similar to that observed in the incisional biopsy, albeit with partially decreased cellularity (Figure 2C, inset), significantly reduced proliferative index (Figure 2D) and areas of intercellular myxoid degeneration. The surgical resection margins were not involved and regional lymph nodes were negative for tumor. Fluorescence in situ hybridization confirmed the ETV6 rearrangement in the residual tumor tissue. The patient's post-operative course was unremarkable and no adjuvant therapy was recommended. The patient has been without evidence of disease with more than 1-year follow-up. 


\section{DISCUSSION}

Histologically, the cellular variant of CMN is very similar to another rare tumor of infancy, infantile fibrosarcoma (IFS), a malignant tumor of fibroblasts. In fact, it is likely that cellular CMN represents a visceral form of IFS[7] (and the classic histiotype, the renal analogue of infantile fibromatosis of soft tissue)[8]. In addition, both tumors share a similar biologic phenotype, with a generally benign clinical course and exquisite sensitivity to chemotherapy. In fact, because both are so highly responsive to chemotherapy, initially unresectable tumors are often pre-treated with neoadjuvant chemotherapy prior to resection. [9] Although most IFS are treated at presentation, spontaneous regression has been reported. Madden et al described a case of a two week old with an IFS in the upper extremity which would have required amputation at the elbow for complete resection. The tumor was observed and by age 7 months the tumor was no longer palpable. By four years of age, the child was without evidence of disease.[10] Ours is the first report to describe partial regression of an untreated cellular variant of $\mathrm{CMN}$, further highlighting the similarity between these two tumor types.

In addition to the histologic and biologic similarities, these tumor types share cytogenetic abnormalities, both being associated with polysomies for chromosomes 8, 11, 17 and 20, $[11,12]$ as well as translocation $\mathrm{t}(12 ; 15)(\mathrm{p} 13 ; \mathrm{q} 25) .[7,13]$ This translocation fuses the ETV6 (also known as TEL) gene on the short arm of chromosome 12 to NTRK3 (also known as TRKC) on the long arm of chromosome 15. This results in an ETV6-NTRK3 gene fusion product which contains the helix-loop-helix protein dimerization domain of ETV6 (aminoterminal end) fused to the kinase domain of NTRK3 (carboxyl-terminal end). [13] ETV6 is a transcription factor originally characterized as an oncogene in several types of leukemias and myeloproliferative syndromes.[14,15] ETV6 translocations can include a number of partners, often tyrosine kinases, such as NTRK3. [14,15] NTRK3 is a member of the neurotrophic tyrosine kinase receptor (NTRK) family of receptor protein tyrosine kinases (PTK) and binds neurotrophin 3 with high affinity, leading to receptor dimerization and autophosphorylation of PTK tyrosine residues.[16] These residues then serve as anchors for downstream signal transduction molecules. The oncogenic mechanism of ETV6-NTRK3 involves ETV6 HLH-mediated dimerization, resulting in constitutive, ligand-independent, NTRK3 tyrosine kinase activity.[17] Thus, dysregulated NTRK3 tyrosine kinase activation, mediated by the ETV6 promoter, is likely important in CMN and IFS oncogenesis, perhaps as the initial transforming event.[7]

As the standard treatment for both tumors is immediate surgical resection, there have been few opportunities to study their natural history. We have presented the case of a child who did not have immediate surgical resection and whose tumor was untreated for 8 months. During that time, the tumor demonstrated significant regression allowing for subsequent complete surgical resection. The contributions of the intercurrent infections and surgical manipulation to the evolution of this tumor are uncertain. Tumor biopsy can lead to hemorrhage, increased intratumoral pressure and subsequent ischemic necrosis, and vasoocclusive changes were seen in the resected specimen. Nevertheless, the shared translocation with IFS, a tumor with well-documented potential for spontaneous regression, suggests that this genetic abnormality may have contributed to the favorable clinical course. The generally benign clinical course and marked chemosensitivty of CMN and IFS is somewhat paradoxical given that the ETV6-NTRK3 chimeric protein has potent transforming activity in NIH3T3 fibroblasts, which then become highly tumorigenic.[18] It has been hypothesized that expression of this oncoprotein somehow renders cells more sensitive to pro-apoptotic stimuli.[9] It is interesting that NTRK3 is also associated with a more favorable prognosis in children with neuroblastoma, another tumor noted for spontaneous regression.[19] 
This case provided a unique set of circumstances that allowed for the observation of the natural history of a case of cellular CMN. Nevertheless, because of rare cases of progression and metastatic spread, observation is probably not the most appropriate course for an infant with a newly diagnosed CMN. However, this case does support the conservative approach to the treatment of these lesions espoused by Beckwith[20] and others given the significant potential toxicities associated with giving chemotherapy to very young patients.[9]

\section{Supplementary Material}

Refer to Web version on PubMed Central for supplementary material.

\section{Acknowledgments}

This work was supported in part by the US Public Health Service Childhood Solid Tumor Program Project Grant No. CA 23099, by Cancer Center Support Grant No. 21766 from the National Cancer Institute, and by the American Lebanese Syrian Associated Charities

\section{References}

1. Glick RD, Hicks MJ, Nuchtern JG, et al. Renal tumors in infants less than 6 months of age. J Pediatr Surg 2004;39:522-525. [PubMed: 15065020]

2. Bolande RP, Brough AJ, Izant RJ Jr. Congenital mesoblastic nephroma of infancy. A report of eight cases and the relationship to Wilms' tumor. Pediatrics 1967;40:272-278. [PubMed: 4378178]

3. Joshi VV, Kasznica J, Walters TR. Atypical mesoblastic nephroma. Pathologic characterization of a potentially aggressive variant of conventional congenital mesoblastic nephroma. Arch Pathol Lab Med 1986;110:100-106. [PubMed: 3004371]

4. Howell CG, Othersen HB, Kiviat NE, et al. Therapy and outcome in 51 children with mesoblastic nephroma: a report of the National Wilms' Tumor Study. J Pediatr Surg 1982;17:826-831. [PubMed: 6298397]

5. Furtwaengler R, Reinhard H, Leuschner I, et al. Mesoblastic nephroma--a report from the Gesellschaft fur Padiatrische Onkologie und Hamatologie (GPOH). Cancer 2006;106:2275-2283. [PubMed: 16596620]

6. van den Heuvel-Eibrink MM, Grundy P, Graf N, et al. Characteristics and survival of 750 children diagnosed with a renal tumor in the first seven months of life: A collaborative study by the SIOP/ GPOH/SFOP, NWTSG, and UKCCSG Wilms tumor study groups. Pediatr Blood Cancer 2008;50:1130-1134. [PubMed: 18095319]

7. Rubin B, Chen C, Morgan T, et al. Congenital mesoblastic nephroma t(12-15) is associated with ETV6-NTRK3 gene fusion: cytogenetic and molecular relationship to congenital (infantile) fibrosarcoma. Am J Pathol 1998;153:1451-1458. [PubMed: 9811336]

8. Argani, P.; Beckwith, JB. Renal neoplasms of childhood. In: Mills, SE., editor. Sternberg's Diagnostic Surgical Pathology. 5. Lippincott Williams and Wilkins; Baltimore Maryland, 21201: 2010. p. 1813

9. McCahon E, Sorensen PH, Davis JH, et al. Non-resectable congenital tumors with the ETV6NTRK3 gene fusion are highly responsive to chemotherapy. Med Pediatr Oncol 2003;40:288-292. [PubMed: 12652616]

10. Madden NP, Spicer RD, Allibone EB, Lewis IJ. Spontaneous regression of neonatal fibrosarcoma. Br J Cancer Suppl 1992;18:S72-S75. [PubMed: 1503930]

11. Schofield DE, Yunis EJ, Fletcher JA. Chromosome aberrations in mesoblastic nephroma. Am J Pathol 1993;143:714-724. [PubMed: 8395771]

12. Mandahl N, Heim S, Rydholm A, et al. Nonrandom numerical chromosome aberrations $(+8,+11$, $+17,+20$ ) in infantile fibrosarcoma. Cancer Genet Cytogenet 1989;40:137-139. [PubMed: 2758397] 
13. Knezevich SR, Garnett MJ, Pysher TJ, et al. ETV6-NTRK3 gene fusions and trisomy 11 establish a histogenetic link between mesoblastic nephroma and congenital fibrosarcoma. Cancer Res 1998;58:5046-5048. [PubMed: 9823307]

14. Papadopoulos P, Ridge SA, Boucher CA, et al. The novel activation of ABL by fusion to an etsrelated gene, TEL. Cancer Res 1995;55:34-38. [PubMed: 7805037]

15. Golub TR, Barker GF, Lovett M, Gilliland DG. Fusion of PDGF receptor beta to a novel ets-like gene, tel, in chronic myelomonocytic leukemia with $\mathrm{t}(5 ; 12)$ chromosomal translocation. Cell 1994;77:307-316. [PubMed: 8168137]

16. Lamballe F, Klein R, Barbacid M. trkC, a new member of the trk family of tyrosine protein kinases, is a receptor for neurotrophin-3. Cell 1991;66:967-979. [PubMed: 1653651]

17. Okuda K, Golub TR, Gilliland DG, Griffin JD. p210BCR/ABL, p190BCR/ABL, and TEL/ABL activate similar signal transduction pathways in hematopoietic cell lines. Oncogene 1996;13:11471152. [PubMed: 8808688]

18. Wai DH, Knezevich SR, Lucas T, et al. The ETV6-NTRK3 gene fusion encodes a chimeric protein tyrosine kinase that transforms NIH3T3 cells. Oncogene 2000;19:906-915. [PubMed: 10702799]

19. Ryden M, Sehgal R, Dominici C, et al. Expression of mRNA for the neurotrophin receptor trkC in neuroblastomas with favourable tumour stage and good prognosis. Br J Cancer 1996;74:773-779. [PubMed: 8795581]

20. Beckwith JB, Weeks DA. Congenital mesoblastic nephroma. When should we worry? Arch Pathol Lab Med 1986;110:98-99. [PubMed: 3004374] 


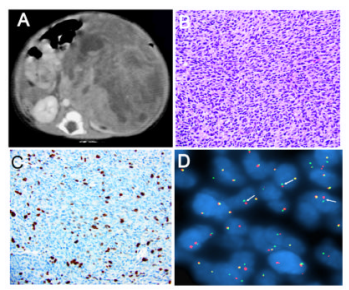

Fig. 1.

(A) Abdominal CT performed on day 1 of life demonstrating a large left renal mass. (B) $\mathrm{H}$ + E staining of a biopsy of the mass (20X). (C) Ki-67 staining of the biopsy specimen (20X). (D) Fluorescence in situ hybridization using an ETV6 DNA probe demonstrating split red and green signals (see arrows), suggesting rearrangement of the EVT6 gene locus. 

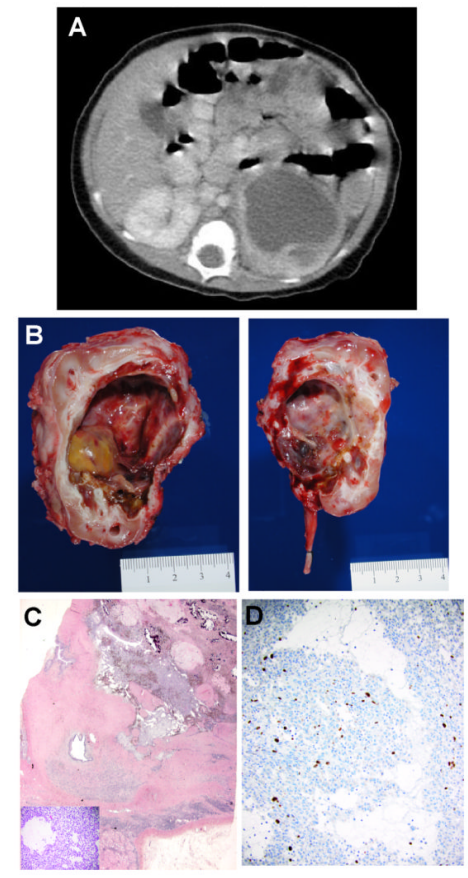

Fig. 2.

(A) Abdominal CT performed at 8 months of age demonstrating significant shrinkage of the left renal tumor with intratumoral cystic degeneration. (B) Gross photographs of the resection specimen. (C) $\mathrm{H}+\mathrm{E}$ staining of the resection specimen demonstrating necrosis, hemosiderosis, calcification and scarring (1X). Also noted was decreased cellularity in the residual viable tumor found at the periphery of the cystic mass (inset, 10X) (D) Ki-67 staining of the resection specimen demonstrating a significantly reduced proliferative index (10X). 